\title{
IdeAs
}

Idées d'Amériques

$17 \mid 2021$

Villes et culture dans les Amériques

\section{Paul Gottfried (dir.) The Vanishing Tradition: Perspectives on American Conservatism}

Ithaca [New York], Cornell University Press (an imprint of Northern

Illinois University Press), 2020, 232 pages

\section{Virgile Lorenzoni}

\section{OpenEdition}

Journals

Édition électronique

URL : https://journals.openedition.org/ideas/10205

DOI : $10.4000 /$ ideas. 10205

ISSN : 1950-5701

Éditeur

Institut des Amériques

\section{Référence électronique}

Virgile Lorenzoni, «Paul Gottfried (dir.) The Vanishing Tradition: Perspectives on American

Conservatism », IdeAs [En ligne], 17 | 2021, mis en ligne le 01 mars 2021, consulté le 04 juin 2021. URL :

http://journals.openedition.org/ideas/10205; DOI : https://doi.org/10.4000/ideas.10205

Ce document a été généré automatiquement le 4 juin 2021.

\section{(c) (†) $\odot$}

IdeAs - Idées d'Amériques est mis à disposition selon les termes de la licence Creative Commons Attribution - Pas d'Utilisation Commerciale - Pas de Modification 4.0 International. 


\title{
Paul Gottfried (dir.) The Vanishing Tradition: Perspectives on American Conservatism
}

\author{
Ithaca [New York], Cornell University Press (an imprint of Northern \\ Illinois University Press), 2020, 232 pages
}

Virgile Lorenzoni

\section{RÉFÉRENCE}

Paul Gottfried (dir.) The Vanishing Tradition: Perspectives on American Conservatism, Ithaca [New York], Cornell University Press (an imprint of Northern Illinois University Press), 2020, 232 pages

1 The Vanishing Tradition, dirigé par Paul Gottfried, universitaire et paléoconservateur, s'ouvre à la fois sur un constat et un objectif : le mouvement conservateur américain produit sa propre histoire, qui «s'éloigne de la réalité factuelle "; il s'agit donc de remettre celle-ci en question. L'originalité de l'ouvrage provient toutefois du fait que, comme le fait remarquer Gottfried en introduction, « la plupart des contributeurs [...] ont un lien quelconque avec la droite, mais pas avec la lucrative opération commerciale qui est associée à ce mot. » Le ton est donc donné, et c'est là l'intérêt principal du livre : le lecteur plonge au cœur d'un conflit idéologique interne à la droite américaine. Gottfried et ses contributeurs prennent le parti de décrire le mouvement conservateur américain comme déconnecté du conservatisme traditionnel, et prêt à tout pour exclure toute voix discordante s'exprimant en son sein.

2 S'il est parfois observé que les écrits sur le conservatisme en tant qu'idéologie émanent des conservateurs eux-mêmes (Müller J., 2006) cet ouvrage se démarque en proposant, plutôt qu'une introspection visant à définir la nature du conservatisme, une véritable remise en question de ce que l'un des contributeurs, Jack Kerwick, appelle le «Big Con » : un mouvement essentiellement dominé par les idées néo-conservatrices, très 
préoccupé par la préservation d'une certaine pureté intellectuelle, et dont le fonctionnement et la promotion reposent sur une puissante infrastructure, dont les garants sont peu enclins à la diversité idéologique.

3 Une telle approche, relativement rare dans la littérature scientifique consacrée au mouvement conservateur américain, permet d'apporter une nuance bienvenue dans la lecture des évènements politiques récents aux États-Unis, notamment en ce qui concerne la compréhension de certains des tenants et des aboutissants de la présidence Trump, dont les développements récents ont parachevé de diviser le Parti républicain. En revanche, on pourrait reprocher à l'ouvrage, dans son attaque contre « l'establishment » néoconservateur américain, de ne représenter le néo-conservatisme que comme un faux conservatisme, qui aurait graduellement usurpé la place du conservatisme traditionnel. Ce faisant, il semble que l'option d'une reconfiguration idéologique soit parfois négligée, au profit d'une simple disqualification du néoconservatisme comme forme valable de conservatisme; un parti pris remis en question par certains spécialistes des idéologies (Freeden M., 1996).

4 L'ouvrage se divise en dix chapitres que l'on peut regrouper autour de trois grands axes. Le premier concerne l'héritage britannique du conservatisme américain ; en effet, la contribution de Grant Havers, par exemple, s'emploie à expliquer dans quelle mesure le néo-conservatisme ne peut revendiquer un tel héritage. Celui-ci s'appuie sur le travail de George Grant, qu'il compare avec les visions de conservateurs américains tels que Frank Meyer, Irving Kristol, ou Russell Kirk. Le chapitre proposé par Jack Kerwick, lui aussi, définit le conservatisme américain comme " rationaliste » et " universaliste », et donc à l'opposé des idées de Burke ou d'Oakeshott.

5 Le second axe concerne la façon dont le néo-conservatisme américain a graduellement expurgé toutes les voix discordantes; on trouve donc, dans les contributions concernées, une analyse du discours et des stratégies utilisés par les néoconservateurs pour discréditer leurs adversaires. Le chapitre de Nicholas W. Drummond, par exemple, entend prouver que la critique néoconservatrice du trumpisme, qui voit en celui-ci une trahison des principes des pères fondateurs, est inexacte, en avançant l'idée qu'elle se base sur une interprétation straussienne qui ignore les « critères anthropologiques du système politique américain ». Le chapitre de Boyd D. Cathey, quant à lui, se penche sur l'exclusion progressive des conservateurs du Sud, notamment en raison d'une adoption graduelle par les néoconservateurs de certaines idées de la gauche multiculturelle, qui poussent à la défiance vis-à-vis de ce que Cathey identifie comme un conservatisme plus traditionnaliste, mais aussi plus identitaire. Presque toutes les contributions consacrées au néo-conservatisme soulignent d'ailleurs le fait que le mouvement est issu de la gauche radicale, comme le chapitre de Keith Preston, qui s'intéresse à «l'affaire M. E. Bradford ", et qui insiste sur l'utilisation de tactiques diffamatoires, que l'auteur associe notamment aux milieux trotskistes, pour empêcher Bradford, un paléoconservateur, d'accéder à la présidence du National Endowment for Humanities en 1980.

6 Un troisième axe, enfin, explore des perspectives plus contemporaines, et met l'accent sur les divisions internes au mouvement conservateur et au Parti républicain accentuées par l'élection et le mandat de Donald Trump. En ce qui concerne la politique étrangère, Marjorie L. Jeffrey compare l'isolationnisme de Trump à celui de Pat Buchanan, lui-même écarté du mouvement conservateur après s'être opposé au « Nouvel Ordre Mondial» de G. H. W. Bush en 1992. Richard T. Marcy s'interroge sur 
l'avenir de l'Alt-Right, en formulant l'hypothèse que la disparition annoncée du mouvement relève probablement du vœu pieux. Un tel chapitre, qui propose une analyse de l'Alt-Right à travers une approche psychologique, prend tout son sens dans le contexte actuel, alors que l'on s'interroge sur l'avenir du trumpisme et de ses partisans les plus radicaux après les évènements survenus au Capitole en janvier dernier. Enfin, George Hawley s'intéresse aux électeurs du Parti républicain, et affirme que la majorité ne sont pas des conservateurs au sens de l'establishment. Là encore, quiconque s'intéresse au futur du trumpisme verra une analyse bienvenue des préoccupations des électeurs, qui, selon l'auteur, ne se retrouvent qu'assez peu dans la vision néoconservatrice proposée par le «Big Con ».

7 L'ouvrage de Gottfried, dont l'épilogue revient sur les purges subies par les conservateurs ne se pliant pas aux idées néoconservatrices, pourrait prendre l'apparence d'une riposte d'une partie de la droite américaine, vouée aux gémonies. Gottfried reconnaît d'ailleurs qu'il a lui-même été la victime des tactiques dénoncées dans l'ouvrage. Cependant, il précise qu'il ne s'agit pas de régler de vieilles querelles, mais de donner la parole à des chercheurs plus jeunes sur un sujet ayant souffert de nombreuses représentations tronquées, tout en « cherchant la controverse ». L'objectif est certainement atteint, puisque The Vanishing Tradition offre un panel de points de vue assez peu représentés dans la littérature scientifique, et permet d'ouvrir des perspectives de réflexion bienvenues pour quiconque s'intéresse au conservatisme américain, à ses mutations idéologiques, et à ses luttes intestines passées et futures.

\section{AUTEURS}

\section{VIRGILE LORENZONI}

Aix Marseille Université, LERMA, Aix-en-Provence, France 